\title{
Mental rotation and the frame of reference in blind and sighted individuals
}

\author{
PATRICIA A. CARPENTER and PETER EISENBERG \\ Carnegie-Mellon University, Pittsburgh, Pennsylvania 15213
}

\begin{abstract}
Mental rotation in the congenitally blind was investigated with a haptic letter-judgment task. Blind subjects and blindfolded, sighted subjects were presented a letter in some orientation between $0^{\circ}$ to $300^{\circ}$ from upright and timed while they judged whether it was a normal or mirror-image letter. Both groups showed an increasing response time with the stimulus's departure from upright; this result was interpreted as reflecting the process of mental rotation. The results for the blind subjects suggest that mental rotation can operate on a spatial representation that does not have any specifically visual components. Further research showed that for the sighted subjects in the haptic task, the orientation of a letter is coded with respect to the position of the hand. Sighted subjects may code the orientation of the letter and then translate this code into a visual representation, or they may use a spatial representation that is not specifically visual.
\end{abstract}

This study examines the characteristics of the spatial information used in mental rotation. One question that is addressed is whether mental rotation requires a visual representation. This issue was explored by examining haptic rotation by blind individuals. Congenitally blind individuals presumably do not have the visual representations available to sighted individuals (cf. Worchel, 1951); consequently, if the mental rotation functions are similar for blind and sighted subjects, it would indicate that visual representations are not necessary for mental rotation. The second part of the paper explores the factors that determine subjective upright in haptic rotation. In particular, for sighted subjects, subjective upright is influenced by the position of the subject's hand.

The original paradigm used to study mental rotation involved two visually presented drawings of cube-like structures. The two figures differed in orientation, and the subject's task was to decide whether the two figures were structurally the same or mirror-images (Shepard \& Metzler, 1971). Response time increased linearly with the angular disparity between the orientations of the two figures, reflecting the mental rotation process. The rotation process also is manifested in the pattern of eye fixations

This research was supported in part by Research Grant NIE-770007 from the National Institute of Education and Grant MH-29617 from the National Institute of Mental Health. We thank the students and teachers, particularly Mrs. Janet Simon, at the School for the Blind Children, Pittsburgh, Pennsylvania, for their cooperation in this research. We also thank Marcel Just for his comments on the manuscript. Reprint requests should be sent to Patricia A. Carpenter, Department of Psychology, CarnegieMellon University, Pittsburgh, Pennsylvania, 15213. P. Eisenberg is now at the University of Minnesota. during the task (Carpenter \& Just, in press; Just \& Carpenter, 1976). Subjects make a series of fixations, looking back and forth between the corresponding features on the two figures, with approximately one additional comparison for every $45^{\circ}$ of angular disparity.

Because of the large visual component in the task, it seems intuitively plausible that the underlying representations are visual. However, an alternative possibility is that the representations are simply spatial. Drawing this distinction requires that spatial and visual representations be differentiated.

A spatial representation contains information about the relative positions of elements or features with respect to some reference coordinates. A visual representation contains this information, but it may also contain additional attributes that are uniquely visual, such as the reflective characteristics of a stimulus, its color, brightness, and visual texture. Moreover, the visual representations contain information associated with the visual sensory experience (cf. Kintsch, 1974; Segal \& Fusella, 1970). For example, if a sighted person visually images a red car, that representation may have as one association a coding of the sensory experience of red cars. The distinction between the two kinds of representations also may have processing implications. The operators that transform a visual representation could differ from the operators that transform a spatial representation.

The underlying representation is not necessarily determined by the modality of the original stimulus information. A visual or spatial representation could be generated from a verbal description, from haptic input, or retrieved from semantic memory. In fact, in a second paradigm used in the study of mental 
rotation, only one figure is visually presented and the other must be retrieved from semantic memory. Cooper and Shepard (1973) presented a single letter like " $y$ " and timed subjects while they decided whether the letter was normal or a mirror image. To perform the task, the subject must retrieve a canonical " $R$ " from memory and compare it to the representation of the stimulus. As predicted, response times increased with the stimulus's departure from upright. Similar processes seem to be evoked in both the single-letter paradigm and in the original paradigm with two visually presented figures.

The first experiment to be reported tested whether visual representations are necessary for mental rotation by comparing mental rotation in congenitally blind subjects and blindfolded, sighted subjects. Congenitally blind individuals cannot generate visual representations as defined previously, although they almost certainly can represent spatial information. To continue with the red car example, if a blind person is asked to image a red car, he may code information about the red color as well as about the car. However, the color representation is probably semantic, a representation of the lexical relations and connotative meanings associated with red, without any representation of the related sensory correlates: However, uniquely visual attributes may be unnecessary for the process of mental rotation. If a spatial representation of an object is sufficient, then the blind subjects may show reaction time functions that are indicative of mental rotation. The experiment examined whether a spatial representation was sufficient input to the mental rotation process and, if so, whether the operating characteristics of the process were similar to those observed for sighted individuals.

Previous research already suggests that blind individuals can perform mental rotation. In a paradigm that was a haptic version of the Shepard and Metzler task, the results for blind subjects differed only quantitatively from those for sighted subjects (Marmor \& Zaback, 1976). Two nonsense shapes were haptically presented to be judged as the same or different. Subjects who were blind from birth had a slower rate of rotation than those who had become blind about age 15; they, in turn, were slower than blindfolded, sighted subjects. In addition, subjects who were blind from birth had a greater intercept, reflecting a longer duration of the encoding or response selection stages. Thus, there is already some evidence that visual imagery is not a necessary prerequisite for mental rotation. While it is possible that sighted subjects in the haptic task still rely on visual imagery, it seems fairly evident that subjects who are blind from birth do not.

The current experiment examines mental rotation in a Cooper and Shepard task, where a single letter is presented haptically in a normal or mirror-image form. This paradigm has several practical features for studying haptic rotation. The stimulus is relatively small, so the subject can quickly locate and encode the discriminating features and begin mentally rotating. Only one figure is presented, so no time is lost trying to find the second figure and to match up corresponding features. If blind individuals give response functions that are similar to those for sighted individuals, it would provide support for the view that mental rotation does not necessarily depend on a visual representation of the stimulus.

\section{EXPERIMENT 1 HAPTIC ROTATION BY BLIND SUBJECTS}

\section{Method}

Subjects were timed while they haptically explored a letter to judge whether it was a normal letter or a mirror image. Two letters, " $P$ " and " $F$," were presented at six orientations, $0^{\circ}$, $60^{\circ}, 120^{\circ}, 180^{\circ}, 240^{\circ}$, and $300^{\circ}$, measured in a clockwise direction from upright. After a trial involving " $F$," the subject would perform one with "P," and so on, alternating through the 24 trials in a block. Thus, the subject knew the identity of the letter before the trial and he only had to determine whether it was normal or a mirror image. The six orientations and normal and mirror versions were presented in a random sequence during each block of trials.

The stimuli were $1 \mathrm{~cm}$ thick and $8 \mathrm{~cm}$ high $\times 4 \mathrm{~cm}$ wide. Each letter was backed with Velcro hook material and firmly attached to disks of Velcro receptor material glued to a presentation board to prevent the subject from physically moving a letter while haptically exploring it. The board was placed in a horizontal position on a table in front of the subject. For half of the subjects, the " $F$ " was always on the left side of the board while the " $P$ " was on the right and the subject moved his right arm and hand to the left or right to reach the " $F$ " and " $P$," respectively. For the other half of the subjects, both letters were presented straight ahead, such that the subject's arm and hand were always parallel to the $0^{\circ}$ orientation on each trial.

The experimenter timed the subject with an electronic timer, initiating it when the subject first touched the stimulus and terminating it when the subject verbally responded either "normal" or "mirror." The subjects were initially given a few practice trials to insure that they understood the directions. The word "rotation" was not used in the instructions. There were six blocks of 24 trials each; the first two blocks served as practice, and only the correct responses from the last four blocks were analyzed. Error trials were repeated within the same block in which they occurred. The subjects were right-handed and used the dominant hand.

The subjects were 12 high school students from Western Pennsylvania School for Blind Children. All 12 were congenitally blind; 5 students reported never having had any visual experience. The other 7 reported having very limited amounts of previous visual experience at some point in their lives; for example, they might have seen light or even had a very limited amount of patterned vision for a short time. Their ages ranged from 15 to 18 years with a mean of 16.8 years.

\section{Results}

Figure 1 shows the latency results as a function of orientation for the blind subjects. The latencies increased with angular deviation from upright, 


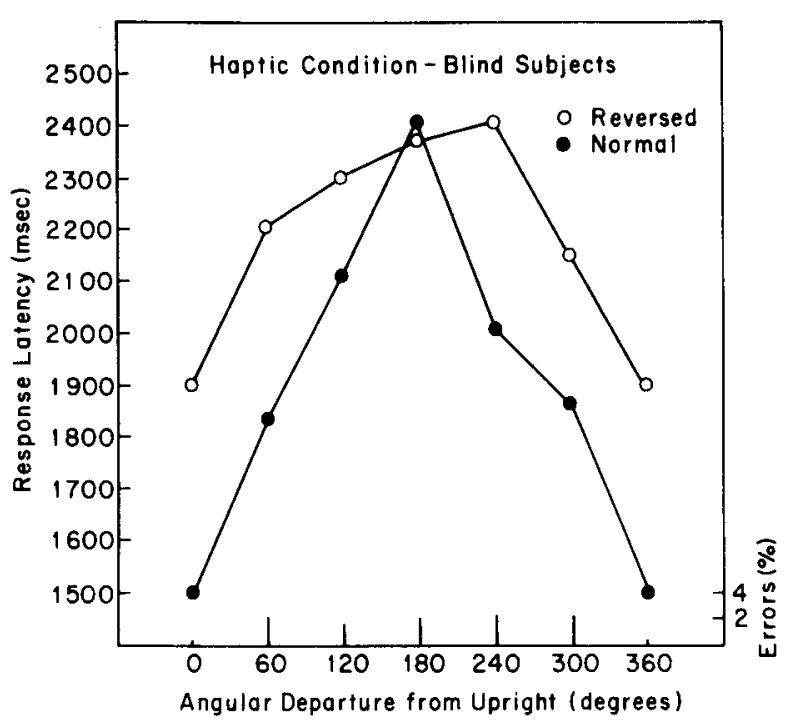

Figure 1. The response latency for the normal and reversed conditions as a function of the letter's angular departure from upright.

$F(5,55)=15.0, p<.01$, with a 690 -msec increase from $0^{\circ}$ to $180^{\circ}$. A trend which increased linearly between $0^{\circ}$ and $180^{\circ}$ and then decreased linearly to $300^{\circ}$ accounted for $94 \%$ of the variance, $F(1,55)=$ $71.92, \mathrm{p}<.01$, and the residual $6 \%$ was not significant, $F(4,55)<1$. (In subsequent experiments, this contrast will be called a linear trend.)

Reversed (mirror-image) letters took $270 \mathrm{msec}$ longer than normal letters, $F(1,11)=9.90, p<.01$. There was a marginal interaction between the normalmirror factor and orientation; the difference between normal and mirror-image conditions was smaller near $0^{\circ}, \mathrm{F}(5,55)=2.12, \mathrm{p}<.10$. No other factors approached significance. The error rate was low $(2 \%)$, and the distribution of errors is shown on the abscissa in Figure 1.

The curves were virtually identical for those subjects who had reported no visual experience and those who reported some small amount of visual experience. There were no statistically significant interactions between these two groups and any other factor. There was also no difference in the results for those subjects who moved their arms to reach the two letters and those whose arms were always straight ahead.

It is interesting to examine the introspective reports of the subjects. The majority claimed to picture the letters as they would draw or "move" them. Subjects who had never seen letters, or had never had pattern visual experience, still claimed to twist the letters around in their minds. This supports the quantitative results that showed no difference between the subjects who had no visual experience and those who had reported some experience. Two subjects claimed to "look for features" and not "move" the letter. For example, an " $F$ " has two short parallel lines on the top right of the stem for an upright letter or on the bottom left of the stem for a letter at $180^{\circ}$. Such a feature code could distinguish normal and mirrorimage figures without rotation. Nevertheless, the 2 subjects who claimed to use such codes had response time functions that were indistinguishable from the other 10.

These results show that mental rotation can operate on a nonvisual representation. In the present experiment, a spatial representation preserves the relative position of certain features. Presumably, this representation must be rotated into an upright form before the subject can judge whether it is the same as a canonical letter or its mirror image.

\section{EXPERIMENT 2 VISUAL CONTROL}

Having established that congenitally blind subjects can perform mental rotation, the issue now turns to a quantitative comparison of the operating characteristics of mental rotation in blind and sighted subjects. To examine the effects of sightedness and the haptic modality, the same task was used with sighted subjects with a visual presentation (Experiment 2) and a haptic presentation (Experiment 3).

\section{Method}

Subjects were timed while they judged whether a visually presented letter was a normal letter or its mirror image. The subject initiated a trial by pressing a "ready" button. Half a second later, the stimulus was presented. The subject's vocal response of either "normal" or "mirror" terminated the trial by activating a relay. Each letter was printed in black on a white index card. The letter subtended $2.5^{\circ}$ of visual angle and was presented in one channel of a two-channel tachistoscope. Otherwise, the design and procedure were identical to that of Experiment 1.

The subjects were 12 sighted college students who participated for course credit. Each session lasted about $\mathbf{4 0} \mathrm{min}$.

\section{Results}

Figure 2 shows the mean response latencies as a function of the angular orientation of the stimulus. The latencies increased $350 \mathrm{msec}$ from $0^{\circ}$ to $180^{\circ}$, $F(5,55)=41.03, p<.01$. As might be expected, latencies in the current task are greater than those obtained by Cooper and Shepard (1973) with more practiced subjects; the mean latency at $0^{\circ}$ is $850 \mathrm{msec}$ in the current task compared with $550 \mathrm{msec}$ in Cooper and Shepard's data. Nevertheless, the overall shapes of the curves are similar; weights derived from the Cooper and Shepard data account for $93 \%$ of the variance among the six orientations, $F(1,55)=191.79$, $\mathrm{p}<.01$, although the residual $7 \%$ is significant, $F(4,55)=3.68, p<.01$. This deviation may be due to the difference between the normal and mirrorimage conditions. Although the normal condition closely follows the function of the Cooper and Shepard data (which is combined across normal and mirror- 


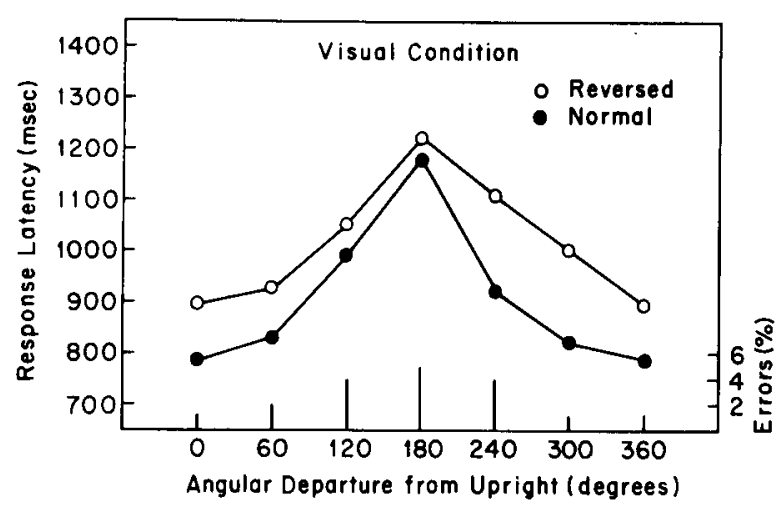

Figure 2. The response latency for the normal and reversed conditions as a function of the letter's angular departure from upright. The letters were visually presented in this experiment.

image conditions), the data for the mirror-image condition in the current task are more linear. Mirrorimage responses took $110 \mathrm{msec}$ longer than normal responses, $F(1,11)=47.63, p<.01$. Moreover, the normal-mirror factor interacted with orientation, $F(5,55)=3.82, p<.01$. As can be seen from Figure 2, the difference between normal and mirrorimage conditions is much smaller when the letter is $180^{\circ}$. No other effects were significant. Errors were a low $3 \%$ overall, and their distribution is shown on the abscissa in Figure 2. This visual condition will provide a baseline for the haptic task with sighted subjects who were blindfolded.

\section{EXPERIMENT 3 HAPTIC ROTATION BY SIGHTED SUBJECTS}

\section{Method}

The materials and experimental procedure for the blindfolded, sighted subjects were identical to that for the blind subjects with one exception. For all subjects, the "F" was presented on the left and the "P" was presented on the right; so the subjects moved their arms to the left and right between successive trials. The subjects were 12 college students who participated for course credit. None had participated in the visual rotation task. The experimental session lasted about $50 \mathrm{~min}$.

\section{Results}

Figure 3 shows the latencies as a function of angular orientation for the sighted subjects. Latencies increased with the letter's deviation from upright, with about a 500 -msec increase in latency from $0^{\circ}$ to $180^{\circ}$ rotation. The overall shape of the curves is similar to the visual control condition in the previous experiment. Weights derived from the visual data accounted for $96 \%$ of the variance among the six means for the orientations between $0^{\circ}$ and $300^{\circ}, \mathrm{F}(1,55)=25.37$, $\mathrm{p}<.01$. The residual $4 \%$ was not significant, $F(4,55)$ $<1$. A linear trend accounted for $87 \%$ of the variance, $F(1,55)=22.98, p<.01$; the residual $13 \%$ was not significant, $F(4,55)<1$. The latencies were longer overall in the current haptic task than in the visual control task. For example, the $0^{\circ}$ orientation produced latencies of about $2,000 \mathrm{msec}$ in the haptic task, compared to $800 \mathrm{msec}$ with the visual presentation.

Latencies were about $160 \mathrm{msec}$ greater for mirrorimage letters than for normal letters, $F(1,11)=10.82$, $\mathrm{p}<.01$. As Figure 3 shows, there was an interaction between orientation and the normal-mirror factor, $F(5,55)=2.64, p<.05$. In this task, the normalmirror differences were smallest near $0^{\circ}$. No other factors approached significance. Errors occurred in about $2 \%$ of the trials, and their distribution is shown on the abscissa in Figure 3.

Overall, the results for the haptic rotation task resemble the results for the visual control, particularly for normal letters (as opposed to their mirror images). The two curves, normal visual and normal haptic, show similar increases in response time as a function of orientation, although they differ in intercept. By contrast, the mirror-image conditions are less similar. The haptic condition shows a particularly large latency increase at $180^{\circ}$.

Two subjects were observed to move their hands during a trial, as though simulating a rotation. One of these subjects claimed that he could not image the figure; he had the most pronounced increase in latency as a function of orientation. The other subject reported that he sometimes mentally rotated the figure and at other times checked for certain features without rotating the figure. There were no obvious differences that discriminated between subjects who reported mentally rotating and subjects who claimed they did not.

There are two interesting differences between the pattern of response times in this experiment and those obtained in Experiment 1 with the blind subjects. First, it should be noted that the blind subjects have

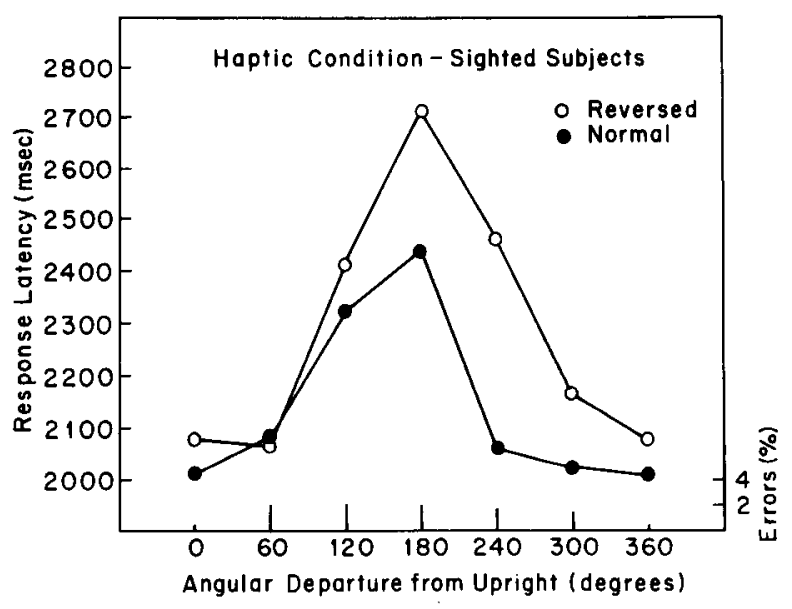

Figure 3. The response latency for the normal and reversed conditions as a function of the letter's angular departure from upright. 
a much lower reaction time for the $0^{\circ}$ stimulus than do the sighted subjects. Hence, it is not always the case that blind subjects show a greater intercept than sighted subjects as Marmor and Zaback found. In the current experiment, most blind subjects had at least some experience with the Optacon, an electronic device that translates print into a tactile stimulus. They also read braille. Hence, they were rather familiar with various haptic tasks. This might explain their faster response time.

Second, the blind subjects showed a linear reaction time as a function of orientation. By contrast, the current results and the visual-presentation results showed a marked curvilinearity. One explanation for the curvilinearity, suggested by Cooper and Shepard (1973), is that letters are often seen in orientations close to upright. ${ }^{1}$ Consequently, we may develop templates or codes for letters that are some degrees from upright. Then it may be sufficient to rotate the representation of the presented letter only up to that critical angle. The value of this critical angle may vary from subject to subject or even from trial to trial, resulting in curvilinearity rather than a step function. Presumably, blind subjects have much less familiarity with letters and do rotate the letters to upright. Consequently, they would not be expected to show curvilinearity.

The curvilinearity in the current task closely resembles the results for the visual task. It might be tempting to ascribe the similar response functions for sighted subjects in the haptic and visual tasks to a similar underlying representation and process. The translation hypothesis could explain the presence of curvilinearity for the sighted subjects and its absence for the blind subjects. Suppose the sighted subject translates the haptic features into a visual representation and the rotation process operates on that visual representation. The curvilinearity in the response time for visually presented letters should be present for haptically presented letters. By contrast, blind individuals could not translate the haptic code into a familiar visual representation; they would not necessarily show the same kind of curvilinearity.

A different explanation might account for some or all of the curvilinearity for the sighted subjects in the haptic condition, if the orientation of a haptic letter is coded relative to the orientation of the subject's hand. Variation in the initial hand position would decrease the overall response time and could introduce curvilinearity. For example, if the subject's hand happened to be oriented at $300^{\circ}$ when a letter was presented at $300^{\circ}$, the subjective disparity would be $0^{\circ}$; by contrast, a letter at $0^{\circ}$ would be coded as having a $60^{\circ}$ disparity, and so on. Suppose the subject's hand orientation varied randomly between $300^{\circ}$ and $60^{\circ}$, then response times for letters at $0^{\circ}, 60^{\circ}$, and $300^{\circ}$ would tend to be equal and shorter than response times for letters at $120^{\circ}, 180^{\circ}$, and $240^{\circ}$. This example shows that in theory the handorientation explanation could account for some of the curvilinearity.

Aspects of the data suggest that the hand position does influence the way a letter's orientation is coded. While the letters " $P$ " and " $F$ " did not interact significantly with orientation, $F(5,55)=1.59$, n.s., there were some differences in their latency functions. The mean latency for " $\mathrm{F}$ " was lowest at $300^{\circ}$ and $0^{\circ}$. Recall that the subject had to reach towards the left side for the "F"; consequently, his right hand would have been oriented close to $300^{\circ}$ with respect to the body's straight-ahead. Conversely, when he reached towards the "P" on the right, his hand's orientation would be close to $60^{\circ}$, and the mean latency for " $P$ "' was lowest at $0^{\circ}$ and $60^{\circ}$. Thus, there is some evidence that hand position might affect the coding of a letter's orientation for these sighted subjects. The next experiment explored this possibility by systematically varying the subject's hand position in a haptic task.

\section{EXPERIMENT 4 THE FRAME OF REFERENCE IN HAPTIC ROTATION}

What is coded as upright in a haptic task could depend on the position of the hand, the position of the body, or some external reference such as the table or floor. To investigate the influence of hand position, the orientation of the subject's hand was varied relative to the subject's body. In one condition, the subject's hand and arm were perpendicular to the subject's frontal plane. In this case, the forearm is parallel to the main axis of a letter at $0^{\circ}$; this is called the "straight" condition. In a second condition, the right forearm was positioned at a $300^{\circ}$ angle to the subject's frontal plane measured in a clockwise direction; this was called the "bent" condition. If the orientation of the letter is coded with reference to the hand position, a letter at $300^{\circ}$ should be coded as upright. A letter at $0^{\circ}$ should be coded as $60^{\circ}$ from upright, and so on. In other words, the response time function for this bent condition should be shifted $60^{\circ}$ from the straight condition. If the letter's orientation is coded relative to some spatial coordinates independent of the hand, there should be no difference between the bent and straight conditions.

\section{Method}

The position of the arm was controlled by putting the subject's forearm between two parallel pieces of wood, $15 \mathrm{~cm}$ high and $30 \mathrm{~cm}$ long, that served as arm guides. Since the two pieces were $9 \mathrm{~cm}$ apart, there was some room for movement. The wrist and hand were free to move. Pilot work had indicated that fastening the wrist itself was impractical. For an entire session, the guide was either in the "straight" or "bent" position. The letters were 
" $F$ " and "R." Otherwise, the design, procedure, and analysis were identical to that in the preceding haptic experiments. Twelve blindfolded, college students participated in the straight condition and 12 in the bent condition.

\section{Results}

Figure 4a shows the results for the straight condition; Figure $4 \mathrm{~b}$ shows the results for the bent condition. The shortest normal response times are at $0^{\circ}$ and $300^{\circ}$ for the straight condition and at $240^{\circ}$ and $300^{\circ}$ in the bent condition. The longest normal response time is at $180^{\circ}$ for the straight condition and at $120^{\circ}$ for the bent condition. The peaks and dips in the response time for the bent condition correspond to what would be expected if the subject's hand orientation determined subjective upright. It appears that for sighted subjects, the frame of reference in haptic rotation depends on the subject's hand position.

The two conditions are very similar, except that the response times for the bent condition are shifted $60^{\circ}$ relative to those for the straight condition. In fact, weights from the straight condition, when adjusted for the $60^{\circ}$ difference, account for $97 \%$ of the variance among the six means in the bent condition, $\mathrm{F}(1,55)=94.48, \mathrm{p}<.01$, and the residual $3 \%$ is not significant, $F(4,55)<1$. By contrast, the weights from the straight condition without any adjustment for the $60^{\circ}$ difference account for only $16 \%$ of the variance among the six means in the bent condition, $\mathrm{F}(1,55)=15.75, \mathrm{p}<.01$, and the residual $84 \%$ is highly significant, $F(4,55)=82.10, \mathrm{p}<.01$. When the initial hand position is taken into account, very similar functions are obtained in the two conditions.

Both conditions show marked curvilinearity. In the straight condition, a linear trend accounted for only $72 \%$ of the variance among the means for the six orientations, $\mathrm{F}(1,55)=33.93, \mathrm{p}<.01$; the residual $28 \%$ was significant, $\mathrm{F}(4,55)=3.33, \mathrm{p}<.05$. In the bent condition, a linear trend (with a minimum at $300^{\circ}$ and a maximum at $120^{\circ}$ ) accounted for only $75 \%$ of the variance, $F(1,55)=73.31, p<.01$; the residual $25 \%$ was significant, $F(4,55)=6.15, p<.01$. Superficially, the curvilinearity did not parallel that in the visual control experiment (Experiment 2). Weights derived from the visual-presentation experiment accounted for only a slightly greater percent of the variance among the six orientations. They accounted for $75 \%$ of the variance in the straight condition, $F(1,55)=34.25, \mathrm{p}<.01$, and $75 \%$ in the bent condition, $F(1,55)=73.31, p<.01$. The residual $25 \%$ was significant, in both the straight and bent conditions, $F(4,55)=2.90, p<.05$, and $F(4,55)$ $=6.15, \mathrm{p}<.01$, respectively. However, the poor fit of the visual-condition predictions was due primarily to two deviations. The first deviation was at $300^{\circ}$ in the straight condition (and at $240^{\circ}$ in the bent condi-

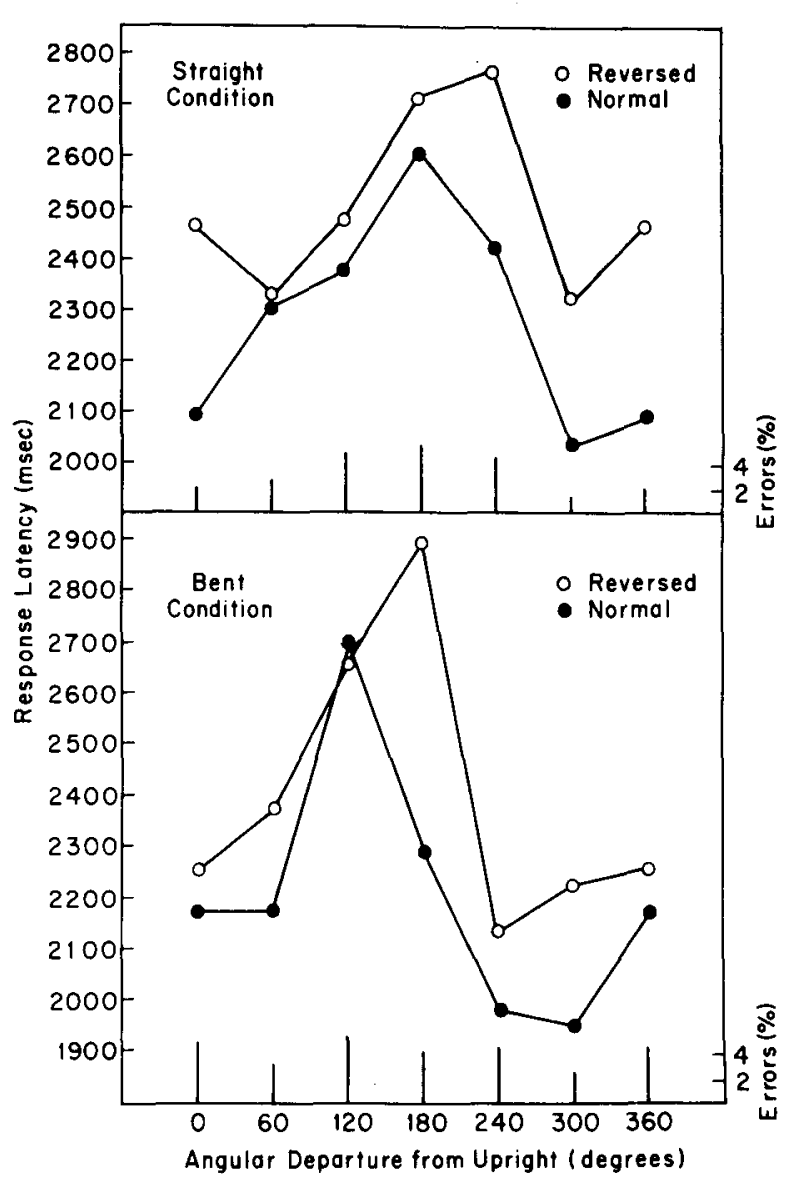

Figure 4. The top panel shows the response times when the arm was in a straight position relative to the subject's frontal plane. The bottom panel shows the response times when the arm was bent to a $300^{\circ}$ clockwise position. The response latencies are shown for the normal and reversed conditions as a function of the letter's angular departure from upright measured with respect to the subject's frontal plane.

tion), where response times were faster than predicted. One post hoc explanation for this may be that the subject is more likely to bend his hand at the wrist, bringing it toward his body rather than away from it. This bias in hand position would reduce the response time for letters whose orientations were $300^{\circ}$ clockwise from the line established by the forearm. The second major deviation occurred for mirror-image responses at $240^{\circ}$ in the straight condition (and $180^{\circ}$ in the bent condition.) The mirror-image responses were unusually long at this orientation, resulting in a significant interaction of orientation and normalmirror judgments, $F(5,55)=2.75, p<.05$, and $F(5,55)=7.19, p<.01$, for the straight and bent conditions, respectively. We have no explanation for this interaction. While the mirror-image data are not parallel to the normal data, the mirror-image data do show a clear effect of hand position. The peaks and troughs of mirror-image responses for the bent con- 
dition are shifted $60^{\circ}$ counterclockwise from those for the straight condition.

There were a number of other effects present. The " $F$ " was responded to faster than the " $R$ " in both conditions, $409 \mathrm{msec}$ faster in the straight condition, $F(1,11)=34.54, p<.01$, and 401 msec faster in the bent condition, $F(1,11)=42.76, p<.01$. Normal responses were significantly faster than responses to mirror-image letters in the straight condition, $F(1,11)$ $=19.67, \mathrm{p}<.01$, and in the bent condition, $F(1,11)$ $=27.01, \mathrm{p}<.01$. In the straight condition, the advantage of the normal response was $400 \mathrm{msec}$ in Block 1 and decreased by about $150 \mathrm{msec}$ in the remaining three blocks, resulting in an interaction of these factors, $F(3,33)=4.08, p<.05$. There was also a significant effect of practice overall in the straight condition; the mean response time for Block 1, 2,611 msec, was slower than the mean response times for the next three blocks, which averaged $2,344 \mathrm{msec}, F(3,33)=8.69, \mathrm{p}<.01$. Of course, subjects had already received two blocks of practice before Block 1. There was no significant practice effect in the bent condition, with the mean of $2,318 \mathrm{msec}$ overall and only a slight, $100 \mathrm{msec}$, decrease in the course of the four blocks, $F(3,33)=$ 1.33 , n.s. No other effects were significant.

These results suggest that at least some of the curvilinearity in the haptic task with blindfolded, sighted subjects (Experiment 3 ) was due to the subjects' initial hand positions. In Experiment 3, hand position was uncontrolled, so the experiment is not directly comparable to either the straight or bent condition of the current experiment. The demonstrated effect of hand position does not rule out the possibility that sighted subjects translate a haptic code into a visual representation. However, the curvilinearity per se is not a simple reflection of the curvilinearity in the visual-judgment task.

An alternative format for representing letters in the haptic task is a haptic representation. Such a representation would contain information about the position of various features, the texture of the object, and the perceptual correlates of the haptic experience. The possibility of a haptic representation cannot be ruled out. However, the general similarity of the reaction time functions for the visual and haptic conditions suggests that the most important determinant of the mental rotation function is the spatial component that is common to the visual and haptic modalities, rather than any uniquely visual or haptic characteristics.

The results for the blind subjects showed none of the curvilinearity found with the sighted subjects. In addition, subjects in Experiment 1 who had to move their hands to reach a figure did not differ from those whose arms were straight ahead. There are two pos- sible interpretations of these results. One possibility is that blind subjects used a more constant hand position than the sighted subjects. However, such a difference between the two groups was not obvious to the experimenter. Another possibility is that blind individuals in Experiment 1 used a frame of reference other than the hand, perhaps the body or cues on the table or in the room. While this hypothesis requires further exploration, it is plausible. Blind subjects may learn to code haptic material independently of hand position, as a result of their greater experience with haptic material in reading braille and in using the Optacon.

A frame of reference may have implications for the order in which features are checked, as well as implications for the orientation assigned to those features. For example, top-to-bottom scanning is a prominent feature of visual inspection in the vertical direction (cf. Ghent, 1961). Such a scanning pattern may be relative to the frame of reference, so that figures that are upright with respect to their frame of reference are scanned top to bottom. Consequently, the time to find a critical feature might depend upon the scanning pattern as well as the feature's location. In the current task, the time to physically locate a feature was probably minimal, since the entire figure was covered by the subject's hand. More likely, the major effect of the frame of reference is on the orientation code assigned to the various features. A feature that is upright with respect to the frame of reference is coded as "upright," even though it would have a very different orientation if coded with respect to some other frame of reference. The coded orientation then determines the amount of mental rotation necessary to bring the representation into congruence with the representation of the canonical letter.

The frame of reference in mental rotation has also been explored for visually presented material by having subjects hold their heads in a normal, upright position or tilted to the left or right (Corballis \& Roldan, 1975; Corballis, Zbrodoff, \& Roldan, 1976). When the stimuli were alphanumeric characters, such as in Experiment 2, the orientation of the head did not influence the pattern of response times. In a second task, with unfamiliar dot patterns that were either symmetrical or asymmetrical about a line, head position did influence the speed with which the observer could judge symmetry. In other words, retinal upright was the frame of reference in this second task. The two different results suggest that the frame of reference in a visual task may depend on the familiarity of the stimuli or on the task, or on both. Finally, instructions to consider the head's position as "upright" will also cause a subject to use a retinal frame of reference (Attneave \& Olson, 1967; Attneave \& Reid, 1968). 


\section{GENERAL DISCUSSION}

These results indicate that visual abilities are not a necessary prerequisite for mental rotation. Blind individuals had no difficulties performing the mental rotation task. Hence, mental rotation is an operation that requires a representation with spatial components rather than specifically visual components. The question still remains as to whether sighted subjects do use representations that include visual attributes. More generally, the same distinction can be investigated in a number of domains. Mental rotation is but one member of a class of operations that process spatial information; other operations include both rigid and nonrigid transformations, such as translation, size scaling, shearing, erosion, etc. In each case, it should be possible to distinguish whether the process operates on representations that are spatial or whether the representation includes additional attributes that are uniquely associated with some modality, be it visual or haptic.

\section{REFERENCES}

Attneave, F., \& Olson, R. K. Discriminability of stimuli varying in physical and retinal orientation. Journal of Experimental Psychology, 1967, 74, 149-157.

ATtNeAve, F., \& ReID, K. W. Voluntary control of frame of reference and slope equivalence under head rotation. Journal of Experimental Psychology, 1968, 78, 153-159.

Carpenter, P. A., \& Just, M. A. Eye fixations during mental rotation. In J. Senders, R. Monty, \& D. Fisher (Eds.), Eye movements and the higher psychological functions. Hillsdale, N.J: Erlbaum, in press.
Cooper, L. A., \& Shepard, R. N. Chronometric studies of the rotation of mental images. In W. G. Chase (Ed.), Visual information processing. New York: Academic Press, 1973.

Corballis, M. C., \& Roldan, C. E. Detection of symmetry as a function of angular orientation. Journal of Experimental Psychology: Human Perception and Performance, 1975, 1 , 221-230.

Corballis, M. C., Zarodoff, J., \& Roldan, C. E. What's up in mental rotation? Perception \& Psychophysics, 1976, 19. 525-530.

Ghent, L. Form and its orientation: A child's eye view. American Journal of Psychology, 1961, 74, 177-190.

Just, M. A., \& Carpenter, P. A. Eye fixations and cognitive processes. Cognitive Psychology, 1976, 8, 441-480.

KINTsCH, W. The representation of meaning in memory. Hillsdale, N.J: Erlbaum, 1974.

MARMOR, G. S., \& ZABACK, L. A. Mental rotation by the blind: Does mental rotation depend on visual imagery? Journal of Experimental Psychology: Human Perception and Performance, 1976, 2, 515-521.

Segal, S. J., \& Fusella, V. Influence of imaged pictures and sounds on detection of auditory and visual signals. Journal of Experimental Psychology, 1970, 83, 458-464.

ShePard, R., \& Metzler, J. Mental rotation of three-dimensional objects. Science, 1971, 171, 701-703.

Worchel, P. Space perception and orientation in the blind. Psychological Monographs, 1951, 65, 1.28.

\section{NOTE}

1. Cooper and Shepard suggest a number of mechanisms that might account for the curvilinearity. For example, letters might be rotated faster as they approach upright. Most of these explanations rely on the fact that letters are familiar in the upright orientations and orientations close to upright.

(Received for publication May 9, 1977; revision accepted November 21,1977 .) 